\title{
Planning Future Strategies for Domestic and International NeuroAIDS Research, July 24-25, 2008
}

\author{
Jeymohan Joseph • David Clifford • Steven D. Douglas • \\ Howard Fox • Howard E. Gendelman • \\ Francisco Gonzalez-Scarano • Igor Grant • \\ Eugene Major • Justin McArthur • \\ the NeuroAIDS Research Participants
}

Received: 22 April 2009 / Accepted: 26 April 2009 /Published online: 20 May 2009

(C) The Author(s) 2009. This article is published with open access at Springerlink.com

\begin{abstract}
The National Institute of Mental Health in cooperation with the National Institute on Drug Abuse and the National Institute of Neurological Disorders and Stroke organized a meeting on July 24-25, 2008 to develop novel research directions for neuroAIDS research. The deliberations of this meeting are outlined in this brief report. Several critical research areas in neuroAIDS were identified as areas of emphasis. Opportunities for collaborations between large NIH-funded projects were also discussed.
\end{abstract}

Keywords neuroAIDS · HIV neuropathogenesis . antiretroviral therapy $\cdot$ drug abuse $\cdot$ neuroinflammation

NeuroAIDS Research Participants. Cristian Achim, University of California, San Diego, San Diego, CA; Sunil Ahuja, University of Texas Health Science Center at San Antonio, San Antonio, TX; James Becker, University of Pittsburgh Medical School, Pittsburgh, PA; Bruce Brew, University of New South Wales, Sydney, Australia; Janice Clements, Johns Hopkins University, Baltimore, MD; Ronald Ellis, University of California, San Diego, San Diego, CA; Leon Epstein, Northwestern University Feinberg School of Medicine, Chicago, IL; Lynda Erinoff, National Institute on Drug Abuse, National Institutes of Health, Bethesda, MD; Dana Gabuzda, Dana Farber Cancer Institute, Harvard Medical School Boston, MA; Harris Gelbard, University of Rochester Medical Center School of Medicine, Rochester, NY; Benjamin Gelman, University of Texas Galveston, TX; David Goldstein, Duke University, Durham, NC; Colin Hall, University of North Carolina, Chapel Hill, NC; Rohan Hazra, National Institute of Child Health and Human Development, National Institutes of Health, Bethesda, MD; Robert Heaton, University of California, San Diego, San Diego, CA; Robin Huebner, Division of AIDS, National Institute of Allergy and Infectious Diseases, National Institute of Health, Bethesda, MD; Kamel Khalili, Temple University, Philadelphia, PA; Dennis Kolson, University of Pennsylvania, Philadelphia PA; Diane Lawrence, National Institute on Drug Abuse, National Institutes of Health, Bethesda, MD; Scott Letendre, University of California, San Diego, San Diego, CA; Thomas Marcotte, University of California, San Diego, San Diego, CA; Michael McGrath, University of California, San Francisco, San Francisco, CA; Susan Morgello, Mount Sinai Medical

\section{Introduction}

A meeting was held to plan future strategies for Domestic and International NeuroAIDS research in Bethesda, Maryland on July 24-25, 2008. This meeting was organized by the National Institute of Mental Health (NIMH) with full cooperation of the National Institute on Drug Abuse (NIDA) and National Institute of Neurological Disorders and Stroke (NINDS). The focus of the meeting was toward developing novel research directions for domestic and international neuroAIDS research. Introductions were made by Dr. Ellen Stover (NIMH) who welcomed participants

Center, New York, NY; Avindra Nath, Johns Hopkins University, Baltimore, MD; Vinayaka Prasad, Albert Einstein School of Medicine, Bronx, NY; Richard Price, University of California, San Francisco, San Francisco, CA; Lynn Pulliam, University of California, San Francisco, San Francisco, CA; Dianne Rausch, HIV Pathogenesis,

Neuropsychiatry and Treatment Branch, Center for Mental Health Research on AIDS, National Institute of Mental Health, National Institutes of Health Bethesda, MD; Kevin Robertson, University of North Carolina at Chapel Hill, Chapel Hill, NC; Ned Sacktor, The Johns Hopkins University, Baltimore, MD; Gerald Sharp Division of AIDS, National Institute of Allergy and Infectious Diseases, National Institutes of Health, Bethesda, MD; Elyse Singer, University of California, Los Angeles, Los Angeles, CA; Stephen Spector, University of California, San Diego, LaJolla, CA; Beth Stevens, Harvard Medical School, Children's Hospital, Kirby Neurobiology Center, Boston, MA; Mario Stevenson, University of Massachusetts Medical Center, Worcester, MA; Ellen Stover, National Institute of Mental Health, National Institutes of Health, Bethesda, MD; Ronald Swanstrom, University of North Carolina at Chapel Hill-Chapel Hill, NC; Victor Valcour, University of California, San Francisco, San Francisco, CA; David Volsky, Columbia University Medical Center, New York, NY; Brian Wigdahl, Drexel University College of Medicine, Philadelphia, PA; May Wong, National Institute of Neurological Disorders and Stroke, National Institutes of Health, Bethesda, MD; Constantin Yiannoutsos, Indiana University, Indianapolis, IN; Christine Zink, The Johns Hopkins University, Baltimore, MD 
and announced the appointment of Dr. Richard Nakamura as the new Director of the NIMH Intramural Research Program and the promotion of Dr. Jeymohan Joseph to Chief of the HIV Pathogenesis, Neuropsychiatry and Treatment Branch at NIMH.

Twenty-three years after its first involvement in acquired immunodeficiency virus (AIDS) research, NIMH now holds a portfolio of 120 grants and two contracts in neuroAIDS (as of July 2008). Despite the years and the numbers of advancements in science and technologies, questions remain and several are fundamental to what it faced 23 years ago. Dr. Stover noted the importance of cooperation and collaboration between institutes including NIDA and NINDS that cosponsored the present workshop. Dr. Robert Eisinger (Office of AIDS Research [OAR]) explained its role in strategic planning for the overall NIH research agenda and budget on HIV/AIDS. The current priorities of this effort are prevention and treatment. OAR welcomed the input and recommendations of the neuroAIDS community. Dr. Lynda Erinoff(NIDA) noted that her agency's neuroAIDS portfolio is under active development. NIDA is working hard with its sister agencies and the research community to enhance its presence in this area of research. Dr. May Wong (NINDS) said her institute is also working to develop a stronger research program in neuroAIDS, and she looks forward to a fruitful discussion in the next 2 days.

Dr. Joseph (NIMH) greeted the participants and acknowledged the help of NIMH's sister agencies in organizing the workshop. The goal of the workshop is to identify key priorities in neuroAIDS research for the next few years with particular emphasis on emerging topics in pathogenesis and new directions for therapeutics. The current research portfolio includes both investigator-initiated research and research projects developed at the program level, such as the CNS HIV Antiretroviral Therapy Effects Research (CHARTER) Program. The workshop is building from ongoing domestic and international research efforts. Discussions are aimed at establishing priorities for neuroAIDS research for future years.

\section{Mechanisms of HIV-SIV neuropathogenesis: emerging research areas}

Dr. Janice Clements (Johns Hopkins University [JHU]) presented data that targeted the response to early, acute lentiviral infection and subsequent control over the course of central nervous system (CNS) disease. Ten years of experiments using pigtailed macaques as a non-human primate (NHP) model of human immunodeficiency virus type one (HIV-1) infection, including serial sacrifice as early as 4 days to trace the precise course of the disease, showed animals that handle the infection better tend to have a higher viral load during acute infection than animals that go on to develop CNS disease. Mechanisms develop in the CNS during the first 7 to 21 days that allow some subjects' brains to deal with both acute and chronic infection more effectively. Markers for subsequent CNS disease include elevated levels of CCL2 in cerebrospinal fluid (CSF) relative to plasma, as well as tumor necrosis factor alpha (TNF- $\alpha$ ) and interleukin-10 (IL-10) in brain tissue. Viral loads in both CSF and plasma are higher in animals that go on to develop CNS disease; but at present, we do not know whether progression is due to the role of virus or the result of inflammatory dysregulation. Researchers need better markers of immune dysregulation and the Hopkins
J. Joseph $(\bowtie)$

HIV Pathogenesis, Neuropsychiatry and Treatment Branch,

Center for Mental Health Research on AIDS,

National Institute of Mental Health, National Institutes of Health,

Room 6202, MSC 9619, 6001 Executive Boulevard,

Bethesda, MD 20892, USA

e-mail: jjeymoha@mail.nih.gov

D. Clifford

Department of Neurology, Washington University,

St. Louis, MO 63110, USA

\section{S. D. Douglas}

Department of Pediatrics and Microbiology,

University of Pennsylvania,

Philadelphia, PA 19104, USA

H. Fox $\cdot$ H. E. Gendelman

Pharmacology and Experimental Neuroscience,

University of Nebraska Medical Center,

Omaha, NE 68198, USA

\section{F. Gonzalez-Scarano \\ Department of Neurology, University of Pennsylvania, Philadelphia, PA 19104, USA}

I. Grant

Department of Psychiatry, University of California-San Diego, San Diego, CA 92103, USA

\section{E. Major}

Laboratory of Molecular Medicine and Neuroscience, National Institute of Neurological Disorders and Stroke, National Institutes of Health,

Bethesda, MD 20892, USA

\section{J. McArthur}

Department of Neurology, Johns Hopkins University, Baltimore, MD 20205, USA 
Group has been looking at both MxA (a downstream gene activated by HIV infection) and interferon beta (IFN- $\beta$ ), which turns on over 100 genes and thus contributes to the immune cascade. It appears that IFN- $\beta$ inhibits viral replication and can even induce dormancy, but only up to a point; and if the brain loses control of the IFN- $\beta$ response (e.g., in response to viral RNA), the virus can begin replicating again, causing a second wave of virus that contributes to later disease.

Dr. Clements identified a number of strategic priorities for the field, many of them related to either a better understanding of past breakthroughs, as well as (or in place of) a better understanding through multidisciplinary approaches. Broader and more collaborative approaches are required because of the unique immunological environment of the brain. NIH could encourage these approaches through grants that span institutes. The field also recognizes the current problems with neuroAIDS in the antiretroviral therapy (ART) era and the subsequent research needs by focusing on ways to protect the nervous system. The field would benefit from further development and exploitation of animal models, especially the macaque model of HIV pathogenesis.

Other important research questions put forward in this lecture included the interplay between innate and adaptive immune responses in the brain as they affect the neuropathogenesis of HIV-1 infection; the impact of ART on disease; bioimaging and peripheral biomarkers as they affect the tempo and progression of disease; adjunctive therapies for neuroAIDS; and virologic and immune responses as they affect the brain including immunosuppression, immune exhaustion, and epigenetics of neuroAIDS.

\section{Host genetic factors regulating HIV-CNS disease: new research opportunities}

Dr. Sunil Ahuja (University of Texas Health Science Center at San Antonio) described a "nodal" model of HIV/acquired immunodeficiency virus, one in which the broad phenotype of "AIDS" reflects progression to specific AIDS-defining illnesses such as HIV-associated dementia (HAD) or cancer. Hence, in genetic epidemiologic studies, it will be important to define strategies to differentiate which genes or genetic pathways associate specifically with HAD versus other AIDSdefining diseases. Additionally, the real challenge is to find genes that have a mechanistic connection with AIDS and not just a correlative connection. Another hypothesis worth consideration in genetic epidemiologic studies is that complex phenotypes are not unique entities, but in fact, are mosaics of common disease-specific alleles and non-disease-specific modifying alleles in the population influenced by a vast array of environmental factors. The latter describes the premise of the common variants/multiple disease hypothesis of common complex genetic disorders, which has been well studied in autoimmune diseases.

The following vignettes highlight these points. First, chemoattraction of monocytes to the brain as well as monocyte/microglial activation is thought to play a key role in the pathogenesis of HAD. To examine this using a genetic epidemiologic approach, Dr. Ahuja's group examined a polymorphism in the gene encoding monocyte chemoattractant protein-1 (MCP-1) which is a potent monocyte chemoattractant; this polymorphism is strongly associated with increased expression of MCP-1. Among HIV-positive persons of European descent, this polymorphism in MCP-1 was associated with an increased risk of both Mycobacterium avium complex (MAC) and HAD. Second, polymorphisms in the apolipoprotein E (apoE) gene are known to play a role in Alzheimer's disease (AD). On the basis of the common variants/multiple disease hypothesis of common complex genetic disorders, it was hypothesized that these polymorphisms may also influence HAD. However, the results showed that the recombinant protein of the APOE isoform that is associated with increased AD risk (APOE3) is associated with increased HIV cell entry in vitro and the ApoE3 genotype was associated with a faster rate of progression to AIDS. But interestingly, an association with HAD was not detected in the cohort studied, which may in part relate to the younger subjects studied.

Other studies were presented illustrating how genetic studies can be used to evaluate mechanisms underlying HIV pathogenesis. For example, delayed type hypersensitivity (DTH) skin test to the neoantigen keyhole limpet hemocyanin was evaluated in HIV-negative subjects. CCR5 genotypes associated with variable DTH responses, but interestingly, those CCR5 genotypes that associated with decreased DTH responses in HIV-negative subjects, predicted a faster rate of disease progression in HIV-positive subjects. These data suggested that CCR5 genotypes may influence HIV-AIDS susceptibility by impacting on viral entry-independent parameters, in addition to their well-described impact on viral entry. Finally, studies were presented showing the association of a genotype in Duffy antigen receptor for chemokines (DARC) with HIV disease course and HAD; interestingly, this genotype is African-specific and associated with resistance to malaria.

In response to questions, Dr. Ahuja posited that some genetic variants might convey immune suppression, and specific alleles may drive disease pathogenesis towards specific AIDS-defining illnesses such as HAD. Some alleles that play a beneficial role in immune depletion may not necessarily have a beneficial effect during immune reconstitution as was shown for some HLA alleles. In addition to DARC, other G-protein-coupled receptors called decoy 
receptors also bind to chemokines without transducing signals into the cell (e.g., D6 receptor).

\section{Novel genomic approaches to delineate host genetic factors involved in susceptibility to HIV-associated dementias}

Dr. David Goldstein (Duke University) explained several, newer approaches that broaden research pursuits for host genetic factors beyond previously studied factors (e.g., apoE, CCR5, or sodium channels). His team has looked more broadly at the entire genome. These approaches were made possible by newer technology such as the Sentrix Human-Hap550 Genotyping BeadChip, which can evaluate 555,000 single nucleotide polymorphisms (SNPs) simultaneously. Because some of the SNPs serve as proxies for others, this effectively scans most of the genome and provides greater power in association studies. This technology does a better job on the European genome than with those who descend from Africa, but it provides a valuable complement to the candidate approach and has already turned up some surprises. Duke's Center for HIV/AIDS Vaccine Immunology (CHAVI) is using the Hap550 to analyze a 600 -sample cohort of HIV-exposed European and Australian patients in order to identify gene variants that play a role in variation in viral set point and progression to $\mathrm{CD}^{+} \mathrm{T}$ cells of $<350$. Raw data suggest that as many as 280 genes affect these endpoints, but two of the strongest associations with viral set point have been the HLA-B 5701 allele and a second SNP located near the HLA-C gene, which together explain $15 \%$ of the variation in set point during early infection. Time to HIV disease progression is also associated with two different genes, one of which (ZNRD1) encodes an RNA polymerase I subunit. The large cohort for these analyses provides good coverage, and the results show that the real benefit of protective alleles can extend time to CD4 loss from 4 to 8 years. This would allow physicians to have a better idea of prognosis, even at diagnosis. However, no such signals have been discovered in African or African-American populations.

The next study using this approach will be in hemophilia patients who have been exposed to HIV, but are not yet infected. In the future, it may be possible to use these techniques to merge the study of HIV and dementia, two genetically and biologically complex diseases that may, nevertheless, have some common factors, as well as many non-overlapping factors.

In response to questions, Dr. Goldstein said that cohort size is a challenge, as can controlling for age, toxicity, and other factors. Many of the 280 "hits" may turn out to be false positives. He believes that next generation chips, in combination with imputation techniques, can get at $80 \%$ of the variation in African populations.

\section{Viral genetics and HIV-CNS disease}

Dr. Dana Gabuzda (Harvard Medical School) outlined the importance of viral diversity seen within clades, with regards to neurotropism, neuroinvasiveness, and neurovirulence. In these works, the hypothesis put forward is that HIV enters the brain during acute infection but remains dormant until the late stages of disease. During this latency, the virus evolves and genetic variations are common in the brain, which reflect founder diversities, selective adaptations to CNS target cells, and immune selection pressure. One HIV-1 gp120 Env variant, N283, gives the virus a preference for cells expressing low levels of CD4, such as macrophages. Sequencing has shown significantly higher levels of the N283 Env variant in brain tissue, compared with lymphoid tissue, and in patients with dementia compared with those with no cognitive dysfunction. Further studies have shown that the loss of an N-linked glycosylation site at position 386 in the HIV envelope V4 region is also associated with dementia. Further study will be needed to determine whether neurotropic variants evolve in the brain and whether therapeutics targeting Env-CD4 interactions will be beneficial in treating HIV-associated neurological disorders.

Differences in neurotropism and neurovirulence can also be seen between HIV-1 clades. In Uganda, for example, subtype D is associated with increased risk of dementia compared with subtype A. There are conflicting findings for clades $\mathrm{C}$ and $\mathrm{B}$, probably because of a lack of understanding of the determinants of dementia. Dual-tropic R5X4 HIV-1 strains, which may evolve after infection, are also implicated in dementia and may be more common than is widely believed. But leaky gut, which elevates the level of plasma lipopolysaccharide, has also been associated with cognitive impairment, and the activation of monocytes may also play a role in CNS infection and viral persistence.

Future studies should include how ART affects viral neuropathogenesis; the relative contributions of viral replication, immune activation, and drug toxicity for brain disease; the impact of clade diversity on neurocognitive impairment; the influence of co-infections, drug abuse, malnutrition, and other confounders; clade-specific difference in drug resistance mutations; and therapies to prevent or ameliorate infection of the nervous system and neurocognitive disorders. 
Therapeutic strategies for HIV-associated CNS disease: current and future trends

Dr. Howard E. Gendelman [University of Nebraska Medical Center (UNMC)] suggested that the field now has validated parameters for ART, including when to initiate, what drugs to use, how to monitor, when to switch medicines, and what drugs to switch. Combinations of drugs work better now and longer than seen previously, and patients benefit demonstrably from the magnitude and duration of viral suppression.

However, ART is very demanding for patients, and nonadherence can lead to therapeutic failures. In addition, drug resistance is a continuing problem. At present, there is a need for improved drug delivery systems. There is also a need for improved biomarkers to guide both diagnosis and therapy, especially for virus-associated neurodegenerative disorders. Such biomarkers might come from transcriptomics (gene chips), proteomics, or metabolomics technologies currently underway in his and other laboratories.

Also needed are adjunctive therapies that can better target, support, or extend the impacts of antiretrovirals (ARV). Particularly attractive would be nanotechnology approaches to formulate and deliver therapeutic agents in nanoparticles. Macrophage-based drug delivery is a promising new technique. Nanoformulated ART essentially manipulates the immune system for therapeutic purposes by using circulating monocytes as drug carriers. The abilities to move drugs inside cells have advantages as a depot for ART and to improve compliance as drugs can be released from cells for periods up to weeks or longer. Testing has been done in humanized mouse models where reconstitution with human stem cells repopulates a deficient immune system. Such models permit studies over months rather than days or weeks. Improved imaging systems, which have evolved from magnetic resonance and computed axial tomography, are being used to track migration of monocyte-macrophages to the brain and target the delivery of specific therapeutics. In response to questions, Dr. Gendelman added that further breakthroughs are needed to optimize nanoparticle uptake and to read the kinetics of drug delivery across the blood-brain barrier.

\section{The classical complement cascade mediates CNS synapse elimination: potential relevance to HIV-associated neurodegeneration}

Dr. Beth Stevens (Harvard Medical School) discussed several similarities in signaling between astrocytes and neurons during neurodevelopment and neurodegenerative disease. In a developing nervous system, immature astro- cytes emit signals that encourage developmental plasticity in the synaptic connections of retinal ganglion cell (RGC) neurons. In the mature brain, astrocytes are silent and RGC nerve connections have been "edited down" to a few unique circuits. It now appears that neurodegenerative disease reactivates the astrocytes, which then re-induce developmental plasticity in neurons. Gene chip analysis shows that astrocytes significantly (10- to 30 -fold) upregulate the $\mathrm{C} 1 \mathrm{q}$ gene in RGC neurons; this same gene initiates the classical complement cascade, whose role is to mediate the elimination of unwanted debris by opsonization (coating) or lysis. Because $\mathrm{Clq}$ tends to localize at the synapses and is developmentally regulated, it thus appears that the cascade is "tagging" unwanted synapses for elimination in the developing brain. This conclusion has been confirmed in a study of C1q knockout mice, which have sustained defects in synapse elimination. This leads to the hypothesis that, in neurodegenerative disease, reactive astrocytes re-express the signal that induces $\mathrm{Clq}$ in neurons, thereby activating the complement cascade to "kill" synapses in the early stages of disease. This hypothesis was tested in the DBA/2J mouse model of human glaucoma, which showed that neurodegeneration was largely $\mathrm{RGC}$-specific, reactive astrocytes are increased in the optic nerve and retina, and $\mathrm{C} 1 \mathrm{q}$ is significantly upregulated at early stages of disease. A similar upregulation of $\mathrm{Clq}$ has also been observed in $\mathrm{AD}$ and other neurodegenerative disorders. These findings are relevant to HAD because $\mathrm{Clq}$ is also upregulated in the early stages of HAD; in fact, HIV gp41 directly interacts with C1q to activate the cascade. Activated astrocytes and other glia are thought to send neuroinflammatory signals, and each astrocyte may touch as many as 100 synapses. Several mechanistic questions remain. What is the signal that activates $\mathrm{Clq}$ ? Which synapses are targeted? How are the "tagged" synapses eliminated? Is this elimination an activity-dependent process? Researchers are trying to develop an in vitro model of synapse elimination, which they believe to be a hypo-activity-dependent "punishment signal." In response to a question, Dr. Stevens said that C1q would be a logical target for molecular intervention if a mechanism could be found to block the signal that activates it. She added that all dementia are complex disorders and that this is, necessarily, a simplistic approach.

\section{Overview of current neuroAIDS research}

Overview of findings from the CNS HIV-1 ART therapy effects research (CHARTER) study

Dr. Igor Grant (University of California at San Diego [UCSD]) presented findings from the ongoing CHARTER 
study, which is gathering data on the relationship between ART and neurologic disease in a cohort of 1,600 HIV-1 seropositive volunteers at six sites. A subset of 600 patients will be followed longitudinally with 6-month follow-ups. At intake, $71 \%$ of volunteers were using at least one ART, with a median of three. Between 2003 and 2007, there emerged several trends in therapy that have had a substantive impact on care and are noteworthy. Drugs containing dideoxynucleosides (D-drugs) declined by $63 \%$; boosting of protease inhibitors (PIs) increased by $40 \%$; overall use of non-nucleoside reverse transcriptase inhibitors (NNRTIs) declined by 24\%, but Atripla increased to $17 \%$ of all regimens; and the proportion of regimens with CNS penetration effectiveness (CPE) scores of $\geq 2.0$ declined by $38 \%$ (increased CPE score is related to viral suppression in CSF). In the CHARTER study, paired CSF and blood samples were collected from 1,247 volunteers. Of these, viral loads exceeded 50 copies $/ \mathrm{mL}$ in $30 \%$ of CSF samples and $52 \%$ of blood samples. CSF values exceeded blood values in $4 \%$, and less than $1 \%$ had detectable loads in CSF, but none in the blood. However, neurocognitive dysfunction is prevalent in the era of ART-about half of the CHARTER volunteers have at least mild cognitive impairment, and the types and degrees of this impairment have changed little with changes in ART. Some neuropathology may be due to co-morbid conditions, but these relationships are complex (past or present drug use is associated with less impairment, while being on ART is associated with more impairment).

Brain neuroimaging studies showed that $34 \%$ of CHARTER volunteers have significant abnormalities in white matter, and MCP-1 (CCL2) emerges as a possible CSF biomarker of white matter injury. White matter abnormalities appear to be dynamic over time, but the volume of abnormalities does not increase in all cases. Investigators are seeking a better classification scheme for lesions. Peripheral neuropathy also remains prevalent in CHARTER volunteers with about $60 \%$ symptomatic. Neuropathy is most common among older patients and current ART recipients, especially those on D-drugs. Drug resistance is a common factor in treatment failure and not all ART drugs penetrate the CNS equally. CHARTER has confirmed earlier findings of "compartmentalized" HIV populations in the CNS and a high frequency of discordant drug resistance patterns between CSF and plasma virus. In a substudy of 185 paired samples, 100 pairs had resistance mutations in one or both compartments, and $39 \%$ of the drug-resistant pairs demonstrated discordant resistance, including 20 volunteers who were currently receiving ART. In fact, current ARV was the strongest correlate of discordant resistance, yet low CPE scores did not appear to be a predictor. Significantly, discordant resistance was positively associated with neurocognitive impairment.
Questions arising from these findings include the following:

- What are the mechanisms for the persistence of neurological disease despite ART compliance and virologic control?

- What is the triggering event?

- How do host genetic factors interplay with viral factors, therapeutics, and co-morbidities to increase the likelihood of neurological disease?

- By what mechanism does discordant resistance relate to neurocognitive impairment?

- How can biomarkers be used to detect and monitor AIDS-related neurological disease?

\section{NIMH-funded international NeuroAIDS efforts (R01 and R21): recent findings and challenges from studies in China, India, Brazil, and Romania}

Dr. Ron Ellis (UCSD) described research on the effects of HIV clade and neurocognitive disorders in Curitiba, Brazil. Whereas all known HIV clades, groups, and subtypes can be found in a central African site like Cameroon, Curitiba has a patient population that is $64 \%$ clade $\mathrm{B}$ and $30 \%$ clade C, making it a natural laboratory for comparing the impact of clade on neurocognitive impairment. The specific aims of this study were to (1) demonstrate the cross-cultural applicability of a neurocognitive assessment that has already been validated in the USA and (2) determine whether neurocognitive impairment is more frequent or severe in Brazilians infected with clade B versus clade C. Volunteers are currently being enrolled in collaboration with the Federal University of Parana and three community organizations in Curitiba.

Dr. Scott Letendre (UCSD) reported on neuroAIDS research in India, where the prevalence of HAD is officially estimated at less than $6 \%$ but may be as high as $56 \%$ with $90 \%$ of patients expressing clade C. It is hypothesized that a variation in clade $\mathrm{C}$ Tat may result in reduced migration across the blood-brain barrier and that the lack of variability in the V3 loop of clade C Env may make it less neurotropic. The research is designed to determine the prevalence and nature of HIV-1-associated neurological disorders (HAND) in clade $\mathrm{C}$ volunteers in India, the impact of ART on HAND and the influence of viral genetics and host factors on the development of HAND in individuals infected with clade $\mathrm{C}$ virus. Researchers will follow 600 volunteers (300 HIV-1 seropositive and 300 controls) in Pune, Maharashtra through initial screening and annual follow-ups; 60 volunteers have been enrolled to date. Challenges include co-morbidities [tuberculosis (TB), malaria, malnutrition], assessment values (illiteracy and low, everyday function), and institutional disconnects that 
have delayed startup. Additional collaborations are planned in northern and southeastern India.

Dr. Cristian Achim (UCSD) described neuroAIDS research in Romania, which has a large population of adolescents who were infected with clade $\mathrm{F}$ as infants. The study will follow 75 volunteers (50 HIV-1 seropositive, 25 uninfected) to confirm the cross-cultural applicability of their neurocognitive test battery, to identify co-morbidities and to characterize the neurotropism of clade F. Preliminary findings indicate that these volunteers face a range of social and medical challenges, with co-morbidities that include hepatitis B and C (HBV, HCV), TB, sub-acute measles and encephalitis. Early samples show a high level of virus in CSF compared with blood, and there is an emerging correlation between stage of HIV and HAND.

Dr. Robert Heaton (UCSD) described two neuroAIDS research projects in China; one in Yunnan (IV drug users) and the other in Anhui (blood donors). In Anhui, researchers enrolled 401 volunteers (203 HIV-1 seropositive, 198 uninfected) and will follow them through 12 annual checkups. At intake, the HIV+ volunteers were mostly CDC stage A, with few co-morbidities other than $\mathrm{HCV}$, and 53\% were already on ART. At 12 months, 95\% of the HIV+ volunteers returned for follow-up and researchers found that $74 \%$ had suffered some neurocognitive decline.

Dr. Vinayaka Prasad (Albert Einstein College of Medicine) reported on a capacity-building program that provides research training for neuroAIDS researchers from India, Rwanda, and Bangladesh. Using the severe combined immuno deficiency mouse HIV encephalitis model, researchers have established that - even when CNS viral loads are similar-infection with HIV clade C causes milder neuropathology and milder cognitive deficits than does infection with HIV clade B. In addition, macrophages infected with clade C recruit fewer monocytes, a subdued response that is mediated by Tat and CCL2, and induce lower levels of chemokines and inflammatory cytokines than clade B. Future research will attempt to translate these findings into human populations, looking at the incidence of dementia associated with progressive HIV-1 infection in clade $\mathrm{C}$ or clade $\mathrm{B}$ populations and determining the mechanism and viral determinants of HAND and HAD, as well as host factors and possible targets for therapeutics.

\section{The International Neurological Study (NIMH-ACTG 5199): recent findings and challenges}

Dr. Kevin Robertson (University of North Carolina at Chapel Hill) reported on the status of this prospective protocol, which is linked by co-enrollment to ACTG 5175 . It will follow 860 treatment-naive volunteers at 11 sites- two each in Brazil, India, Malawi, and South Africa, and one each in Thailand, Peru, and Zimbabwe-who will be given three different ARV combinations and tested using standardized neurocognitive and neuromotor tools. The strengths of this design include consistent follow-up, high retention rates, and shared infrastructure (the AIDS Clinical Trials network). Challenges include language, culture, and network issues, as well as great distances that complicate supply, samples, and data transfer. Future studies may include controls, additional clades and subtypes, and additional treatment regimes.

In response to questions, Dr. Robertson added that informed consent is a widespread and continuing problem. Investigators want a uniform process for institutional review board (IRB) approvals. He discussed the need for the creation of central IRB organizations, which should be part of the international infrastructure. Cultural differences can be so great that US standards and procedures may well be irrelevant in Thailand and elsewhere. The Division of AIDS or DAIDS now has a central person for consent issues, and the National Cancer Institute has regional IRBs, which are useful backup for local groups. Other participants suggested that investigators take advantage of the National Institute of Allergy and Infectious Diseases (NIAID) Networks or similar groups such as INSIGHT, START, ESPRIT, or CPCRA.

\section{NIH-funded resources for domestic and international neuroAIDS research}

Opportunities for collaboration between NeuroAIDS investigators and NIAID- or NICHD-funded projects

Dr. Robin Heubner (NIAID) described the Multicenter AIDS Cohort Study (MACS), which has been enrolling participants at four sites since 1984 and now stands at 7,000 homosexual and bisexual men: 2,500 of them in long-term follow-up (1,200 $\mathrm{HIV}+, 1,300$ uninfected), including $24 \%$ African-American and $11 \%$ Latino. Semiannual visits collect data on 8,500 variables, including confounders and co-morbidities, and the MACS currently holds 1.2 million aliquots of serum and CSF. Neuropsychological screening includes psychosocial questions, standard verbal and motor tests, and a trailmaking task and symbol digit test; volunteers are referred for further testing as appropriate. Investigators do not have to be part of MACS to make use of this resource - they can apply for specimens and data using a simple form, and the program seldom refuses such requests. However, MACS does operate under the "dibs" rule (first come, first served). Outside investigators do well to collaborate with participating investigators.

Dr. James Becker (University of Pittsburgh Medical School), a participating MACS investigator, reported that 
the program is collecting enough data to occupy several research careers. Admittedly, there is a survivor bias-these data represent long-term non-progressors-but outside investigators can obtain clean, case-controlled data on the entire cohort or on subsets, such as stimulant users, without having to do any recruiting themselves. He would like to see MACS extend its data collection to include cerebrovascular disease subsequent to ART and imaging data to differentiate between HAND and co-morbidities.

Dr. Gerald Sharp (NIAID) summarized the Women's Interagency HIV Study (WIHS), launched in 1993 by several $\mathrm{NIH}$ institutes, which is currently following 2,500 women who represent the epidemic in terms of race, ethnicity, drug use, etc. This too is a large, well-characterized cohort with a lot of specimens that has already been the subject of 30 funded studies and has the capacity to rapidly address new avenues of research. Aging and neurocognitive disorders are new areas of emphasis, and investigators have recently added hearing and balance tests to a battery that includes screening for trauma, posttraumatic stress disorder, depression, and menopause. Sharp added that the disconnect between the WIHS site from the sites of HIV clinical care have made some of these measures and outcomes unclear. Future research directions will include a greater emphasis on the influence of behavioral factors on disease progression and HIV management.

Dr. Rohan Hazra (National Institute of Child Health and Human Development [NICHD]) described three pediatric and adolescent HIV cohorts supported by NICHD: (1) Pediatric HIV/AIDS Cohort Study (PHACS) - 34 sites at 24 institutions in 20 US cities, following $400 \mathrm{HIV}-1$ volunteers and 150 controls with semiannual visits that include behavioral and neurological screening; (2) NICHD International Site Development Initiative (NISDI) - 15 sites in eight cities in four Latin American countries, currently enrolling volunteers for two protocols that are similar to PHACS; and (3) Adolescent Medicine Trials Network (ATN) for HIV/AIDS Interventions - 15 sites in the USA that follow behavioral, microbicidal, prophylactic, therapeutic, and vaccine interventions in HIV-infected and HIV at-risk adolescents age 12 through 24 . In response to questions, Dr. Hazra said that at present there is no single catalog of cohorts and specimens (sera, cells, preservation methods) that can be accessed through these networks but that such a catalog would be a valuable resource.

National NeuroAIDS Tissue Consortium (NNTC): future research directions and opportunities for research collaborations with the neuroAIDS community

Dr. Benjamin Gelman (University of Texas) explained that combination ART has improved the health and survival of HIV patients, but neuroAIDS remains prevalent. The mission of NNTC is to collect and distribute to the research community critical biological specimens and biomedical data from patients with HAND, peripheral neuropathy, and other comorbid conditions. This means that it now constitutes a unique, longitudinally characterized group of HIV-infected individuals that is geographically and demographically heterogeneous, includes a relatively old and sick population, and provides a lot of data on complex neurological manifestations. The NNTC cohort currently includes 2,262 volunteers, of whom 2,076 are HIV-1-seropositive and 622 are currently active. Specimen collections include a wide range of CNS and other tissues, including 417 HIV+ brains. Volunteers have also been characterized for prevalence of HAND and peripheral neuropathy, psychiatric and substance abuse disorders, and many histopathology diagnoses.

Shipments of NNTC specimens and data to investigators rose from only 377 in 2002 to 2,427 in 2007, contributing to 241 publications and 11 current grants, but in Gelman's opinion, this collection remains an underutilized resource. NTCC is taking steps to standardize, harmonize, and consolidate data management from contributing sites; and it has plans to install new software tools that will make it easier for investigators to obtain statistical analyses, as well as raw data from the NTCC database. In the future, NTCC brain samples should play a unique and valuable role in comparing, correlating, and confirming the physical and chemical changes that take place in HIV-associated disease at the level of specific circuits and regions of the brain. They will also allow investigators to compare neuropathological outcomes with specific therapies antemortem and to disentangle the contributions of HIV and co-morbidities, particularly HCV and drug abuse.

Despite the many opportunities offered by this resource, limited financial and human resources mean that not all avenues will be pursued. NNTC believes that the following areas of research should be priorities:

- Include a greater portion of aged people, in order to compare normal aging with neurodegenerative aging

- Integrate comorbid conditions into the neuroAIDS agenda

- Pay more attention to HAND of the "other type" (i.e., without HIV-encephalopathy)

- Use NNTC resources to establish and promulgate standards of clinical relevance

- Use NNTC dorsal root ganglia collection to promote study of peripheral neuropathy

- Elucidate the unique attributes of ethnic, linguistic, and educational minorities with HIV

- Use NNTC brains, fluids, and tissues to study CNS virology and HIV evolution in the context of ART

- Study the impact of genetic and epigenetic changes in neuroAIDS

- Standardize, harmonize, and consolidate NNTC with other neuroAIDS databases 
In response to questions, Dr. Gelman added that it is difficult to study $\mathrm{AD}$ and other non-HIV dementia in the NNTC, but there is no evidence that they are more common in HIV-1-seropositive patients. This finding is hardly conclusive; however, since HIV autopsies are age 50-70, they might miss subtle precursors of AD. There is a common worry that HIV accelerates aging, but there is reason to think that HAD would contribute to the clearing of diffuse plaques, not their accumulation. Perhaps, it would be possible to use NNTC samples to validate markers of the early development of Alzheimer's, but perhaps NNTC research should instead focus on subcortical dementia (like HAND, Parkinson's and Huntington's disease) rather than cortical dementia such as Alzheimer's.

NIMH centers program: potential role as a national resource for neuroAIDS research

Dr. Ronald Swanstrom (University of North Carolina at Chapel Hill) explained that the three NIMH-funded centers of neuroAIDS research provide the infrastructure and critical mass of expertise for synergistic approaches, using inter- and multidisciplinary techniques, whose results truly are greater than the sum of their parts.

Dr. Howard Fox (UNMC) described progress in developing biomarkers for HIV infection and neuropathological sequelae. "Omics" technologies, in particular, have facilitated rapid discovery and validation of biomarkers for HAND. Just as genomics has contributed to our understanding of viral and host factors that affect the course of infection, transcriptomics reveals that interferon-induced genes play different roles in blood and brain and that osteopontin can provide a valuable marker for HAND in the ART era, although it is not diagnostic. Proteomics, likewise, has revealed that HIV infection causes multiple changes in blood and CSF proteins preceding the onset of clinical CNS disease. Metabolomics has led to the identification of several low-molecular-weight metabolites that reflect biochemical changes in the CNS following infection. Taken together, these approaches may lead to fast, sensitive tests for diagnosing and monitoring neuroAIDS.

Dr. Grant (UCSD) explained that UCSD's HIV Neurobehavioral Research Center (HNRC) has five research cores that provide resources and opportunities for startup research: neuromedical, neurobehavioral, neuroimaging, neurobiology, and neurovirology. HNRC has recently added an international core, which provides training, technical support, and information exchange for foreign investigators, who receive about $\$ 15,000$ per year to produce preliminary data and assistance in proposal design, and additional money to actually apply for grants. This assistance has led to success for seven different research projects with $\mathrm{NIH}$ funding coming in the form of U13, R21, and R01 grants.
Dr. Justin McArthur (JHU) pointed out that, to date, no therapies, diagnostics, or biomarkers for HAND have entered clinical practice. Therapy in particular remains a serious gap, since HAND remains prevalent even in the ART era, and adherence is known to be a problem in cognitively impaired patients. As a result, Hopkins has established a therapeutics core to provide support for new researchers, innovative studies, and collaborative approaches. Its initial focus is on screening for neuroprotective compounds, but it is also developing a clinical cohort and protocols for clinical trials. There is also a need for simple biomarkers (associative, correlative, or predictive) that are cheap and simple enough to be rolled out in low-resource nations. Plans to build a colony of macaques, and perhaps develop transgenic animals, have not yet been funded. Clearly, there is ample opportunity for interactions and collaborations with other neuroAIDS centers.

\section{Therapeutics}

\section{NIMH Integrated Clinical-Preclinical Program (IPCP)} for novel neuroAIDS therapies

Dr. Steven D. Douglas (University of Pennsylvania School of Medicine) described progress in developing an HIV therapy based on antagonists to the neurokinin-1 receptor (NK-1R). NK-1, also known as substance P, was the first neuropeptide discovered (1931). It is widely distributed in the central and peripheral nervous systems, where it functions as a neurotransmitter, modulator of blood flow and gastric activity, and-importantly - a potent mediator of neuroimmunoregulation. An antagonist to NK-1R (aprepitant) is standard therapy for chemotherapy-induced nausea and vomiting and is also known to have antidepressant activity. In addition, there is known to be a bidirectional interaction between NK-1 and HIV. Serum substance P levels rise significantly following HIV infection, but NK-1R agonists potently inhibit HIV replication in human macrophages, apparently by downregulating CCR5. Four projects were launched to investigate these effects. Using R01 grants from NIMH and tissue samples from NNTC, researchers were able to confirm the in vitro and in vivo antiviral activity of NK-1R. Further research has identified a new, tailless variant that remains on the cell membrane, further inhibiting the infectivity of HIV-1; this variant is the current target of development. Researchers submitted their initial IND application in July 2006; and after FDA comments, a revised IND was approved in September 2006. The trial opened in January 2007 and is currently accruing volunteers. Future directions include further research on mechanisms, as well as 
trials of aprepitant in combination with various boosters and in ART failure.

Dr. Harris Gelbard (University of Rochester Medical Center) summarized a long-term collaboration between the University of Rochester and UNMC, which first determined that HIV Tat enhances the activity of glycogen synthase kinase 3-beta (GSK-3 $\beta$ ), an enzyme that plays a direct role in neuronal apoptosis; identified a mixed lineage kinase type 3 (MLK-3) as an alternative target of Tat; and demonstrated that an inhibitor to MLK-3 can block the toxic effect of Tat and extend the survival of rat and human neurons in vitro. Ironically, this inhibitor (CEP1347) was available from Cephalon Inc., which had developed it as a treatment for Parkinson's disease and did not see neuroAIDS as an important target. Researchers have partnered with BioFocus DPI to further refine CEP1347 to arrive at two lead compounds for preclinical testing. Meanwhile, a related project has demonstrated that CEP1347 also blocks that action of $\mathrm{CPAF}$, another HIV toxin; this suggests a second (neurovascular) locus of protection. Parallel studies have shown that CEP1347 does not interfere with the antiviral efficacy of NRTIs. Phase 1A pharmacokinetic studies are currently underway.

Dr. Michael McGrath (University of California, San Francisco) presented an update on his work in macrophagetargeted drug development for neuroAIDS, in collaboration with Boston College, the University of Hawaii, and Pathological LLC. Discovered in 1898, mitoguazone, also known as MGBG, had been investigated but abandoned as an anticancer drug. However, it was also found to cause the selective loss of CD16+ and CD16+ cells that contain HIV-1 DNA, specifically by blocking the synthesis of osteopontin and Mac387 that are needed to transform a circulating macrophage into a tissue (or CNS) macrophage. MGBG appears to block CNS migration. Simian immunodeficiency virus (SIV)-infected macaques treated with MGBG no longer had infected macrophages in CNS, and the drug appears to kill recent migrants rather than resident cells. However, MGBG did not reduce plasma viremia, although it does appear to shut down viral evolution. Current focus is on the mechanism of operation and additional animal studies, plus the development of biomarkers and clinical trial protocols. In response to questions, Dr. McGrath said that macrophages are a major site of viral evolution, if only because macrophages do not die while replicating virus. However, reservoirs of virus (and evolution) also exist in the meninges, which are relatively understudied. MGBG appears to be safe over the long term, as it has been through 26 cycles of biweekly treatment in patients with AIDS.

Dr. Mario Stevenson (University of Massachusetts Medical Center) explained that because HIV-1 has such a small genome, it must exploit host processes to replicate and spread. However, several cellular restrictions (antago- nists) resist this viral hijacking. For example, APOBEC3G (A3G) restricts viral complementary DNA (cDNA) synthesis and integrity, and tetherin restricts viral release from the cell membrane. Consequently, much of HIV's evolution targets these cellular defenses by producing accessory proteins such as Vif, which binds to A3G and induces its degradation, and $\mathrm{Vpu}$, which counteracts tetherin and permits the release of new virus. Based on this knowledge, investigators hypothesized that it should be possible to identify small molecule antagonists of these and other viral proteins, thereby restoring the protective function of cellular restrictions. They began by screening a library of 30,000 small molecules for Vif inhibitors, leading to the identification of two lead compounds (RN-18 and RN-19) that inhibit Vif with the desired selectivity and effectiveness. Mechanistic studies revealed that RN-18 increases cellular $\mathrm{A} 3 \mathrm{G}$ production in the presence of Vif and increases $\mathrm{A} 3 \mathrm{G}$ incorporation into virions, inducing hypermutation of viral cDNA, confirming that the HIV-1 Vif-A3G axis is a valid target for developing small molecule-based therapies and/or enhancing innate immunity against viruses. The next steps in developing such compounds will be to continue animal studies up through rodents and NHPs and to search for other HIV accessory proteins that might be similarly targeted. Dr. Stevenson closed by noting evidence of fossil viruses in the human genome that are sensitive to similar restrictions; the challenge with HIV is to help the virus become endogenous in less than 30,000 years.

Dr. Avindra Nath (JHU) identified three principal challenges in drug development for HAD and HAND:

1. Identifying targets for treatment. Many different targets have been identified, but most involve protecting the neuron. Relatively few address earlier steps in the HAD pathway, such as viral entry, replication, reservoirs, and glial activation. It is hoped that NIMH's Novel NeuroAIDS Therapies: Integrative Preclinical/Clinical Program (IPCP) will help to expand the search by funding research like the projects described above.

2. Developing therapeutic agents for those targets. Many agents have been tested, most do not work, and none work very well. Drug development requires a library of candidate compounds and a series of increasingly selective high-throughput screens. It has proven to be very complicated (and very time-consuming) to screen a novel library with a functional assay. Here again, IPCP may prove helpful.

3. Collaborating with pharmaceutical companies. IPCP also requires collaboration with industry and this introduces a new set of challenges. Big Pharma has no interest in neuroAIDS. Therefore, investigators must work with small companies, but small companies have short life spans and are subject to disruptive buyouts. This leaves 
university-supported startups, which typically have limited scope and require investment, space, and other resources. Universities can help by providing incubator facilities to support and encourage these startups.

In response to questions, Dr. Nath added that intellectual property can be a problem - generic drugs have no appeal to any industrial partner, large or small; novel compounds are far more attractive. However, the FDA "orphan drug" program has shown that federal support can facilitate the development of drugs and biological products for rare diseases (read, "small markets"), and NIAID has aggressively moved promising drugs into clinical trials. It would be useful to bring these models to NIMH to encourage the development of new therapies for neuroAIDS.

\section{NINDS-funded Neurological Aids Research Consortium (NARC)}

Dr. David Clifford (Washington University School of Medicine) reported that NARC was established in 1993 on the model of the NIAID-funded AIDS Clinical Trials Group (ACTG). NARC serves as a catalyst for leadership and new ideas in what has become a consortium of 30 research centers that conduct multicenter, controlled clinical trials of neuroAIDS interventions, in addition to some independent studies and several collaborations with industry and international partners. NARC's priorities are reflected in its major research areas:

- Peripheral neuropathy-seven major projects in what is the most common clinical complaint in neuroAIDS

- Neurocognitive disorders-eight studies, including three international collaborations

- Opportunistic diseases - four studies of progressive multifocal leukoencephalopathy and other opportunistic diseases of neuroAIDS

- Domestic and international partnerships, including sites in Uganda, Ethiopia, and Malaysia

NARC has always had a great interest in new drugs and their effects on CNS and peripheral disorders. A new study of Paxil (paroxetine) is currently under development, and NARC will also be investigating the effects of multiple drug regimes for peripheral neuropathy. In the future, there are plans for more focused research on neuroregeneration and pain management.

\section{Priority-setting discussion session}

Dr. Dianne Rausch (NIMH) thanked the presenters for their review of the past and present of neuroAIDS research and asked the participants to turn their attention to its future.
NIMH is now doing the strategic planning for initiatives that will be launched in fiscal years 2010 and 2011, and it wants to hear the ideas of this group, which includes most of the senior leadership in the field. NeuroAIDS promises to become an increasingly important topic, and the cohorts and specimen collections described in this workshop represent unique opportunities for collaboration. Funding will remain tight; however, NIMH seeks advice on how to use these resources most efficiently to address the most important targets. With the November election, the nation enters an era of change-political, professional, and medical. How best can NIMH turn these changes into opportunities for neuroAIDS research?

\section{Clinical research}

Dr. Francisco Gonzalez-Scarano (University of Pennsylvania) suggested researchers look at Africa to find both the most pressing questions and their likely answers. Recent evidence points to microbial translocation as the cause of systemic immune activation in chronic HIV infection, and many of the symptoms of neuroAIDS (as well as the opportunistic disease of HIV) look very similar to autoimmune diseases. Yet all current therapies against HIV target the virus (entry, integration, transcription) rather than its effects. In addition, diagnosis for both HIV infection and CNS viremia typically comes too late. Questions to consider include:

- How do we address the problems of the developing world?

- If there are CNS-specific treatments, how do we decide who to treat and when?

- What is the influence of monocyte and T-cell activation on HAD?

- Are minor cognitive problems worth treating or is their genesis too confusing?

Dr. Fox (UNMC) observed that a great deal of work has been done on HIV-related neurocognitive disorders and dementia, but he asked whether this informs us about the treatment of patients as HIV becomes a chronic disease (at least in industrialized countries). Perhaps, we need a new paradigm that focuses on lowering the virus count in the brain and restoring a reasonable immune function. This new paradigm raises new questions. What is the best model of the chronic state under ART? How is CNS immunity different from (and affected by) the rest of the body? What are the patterns of CNS disease (e.g., cortical versus subcortical)? Is HAND a different neuropathological entity from HIVE or HAD? Biomarkers will be vital to the research that will answer these questions, as are better in vitro and in vivo models of neuroAIDS. Other important issues:

- Is neurogenesis impaired and does it matter?

- Neuroprotective responses and/or strategies 
- Co-morbidities

- Immune reconstitution inflammatory syndrome and other treatment effects

- Viral and host genetics

International neuroAIDS research

Dr. Eugene Major (NINDS) reported that NIH spends about \$365 million per year for AIDS research conducted in international settings, out of a total AIDS budget of almost $\$ 3$ billion. However, he felt that only a small proportion of the $\mathrm{NIH}$ NeuroAIDS research budget is spent in international settings. In addition, most of the money for neuroAIDS currently goes to etiology and pathogenesis, with very little going to areas such as vaccines and therapeutics. Nevertheless, there are several areas of basic research in which the nervous system plays a key or unique role:

- Blood-brain barrier-virus, cell trafficking, drugs

- Brain as a reservoir for latency — where, how, and how long

- Neurotrophins - protective and regenerative mechanisms

- Neural stem cells-targets for infection, response to damage

- Neuronal pathology - transmitters, ion channels, plasticity

- NHP models - viral strains, clades, and pathogenesis

Many basic and clinical questions are difficult to address in domestic settings, including natural history, comparative populations, and viral pathology. International research is best pursued through preexisting networks that have cohorts, infrastructure, and community contacts. Better coordination is needed in China and India, where local networks are poorly linked. The time has also come for better coordination across networks in such areas as cohorts, sample inventories, and protocol design.

Dr. Grant (UCSD) presented a broader set of issues that need to be addressed in international neuroAIDS research:

- What can international studies contribute to incremental scientific knowledge about viral and host genetics, cofactors and co-morbidities, and therapeutic regimes?

- NeuroAIDS-relevant measures should be added to ongoing international cohort studies, which can address these questions at a low cost, but perhaps with less sensitivity to early symptoms.

- More complicated questions (e.g., neuropathogenesis, mechanisms) will require well-trained multidisciplinary teams with appropriate infrastructure, such as wellequipped regional centers of excellence.

- Greater international contributions will come only with greater standardization of case ascertainment, diagnosis, laboratory methods, and data management to ensure global relevance, comparability, and generalizability.

- Finally, there is a great need to simplify and harmonize the regulatory processes to improve the timeliness of protocol review by US and foreign authorities; a process that Grant characterized as "bureaucrolysis."

\section{Therapeutics}

Dr. Douglas (University of Pennsylvania School of Medicine) suggested six characteristics that should be met by candidate neuroAIDS therapeutics and regimens: target entry, drugsparing, adjunct to ART, antiinflammatory, neuroprotection, and reservoir depletion. In addition, a prompt way to move candidates through the drug development pipeline needs to be found. One way to do so would be with simulation studies, which could then be confirmed by pharmacokinetics. Finally, we need to agree on a level of acceptable resultswould it be a 3-log reduction in copies per milliliter over 10 days? Even against that standard, however, there might be some interest in a drug like Maraviroc (a CCR5 antagonist), which achieves only a $0.5-1.0 \log$ reduction, but nevertheless is the first therapeutic that targets entry, rather than the virus itself.

Dr. McArthur (JHU) suggested that there remains a need for research on the impact of neuroAIDS and for better biomarkers for diagnosis and treatment response. He also posed a series of clinically relevant questions to be addressed in the design of neuroAIDS therapeutic and adjunctive trials:

- How reversible is HAND, and when should treatment start?

- If virologic suppression is complete, will this protect against HAND?

- What new targets of therapy are most likely to close the treatment gap?

- What are the determinants of treatment response?

- How can we identify co-morbidities, especially aging and depression?

- Can we improve outcome measures to reduce variability and sample size?

Pediatric neuroAIDS

Dr. Leon Epstein (Northwestern University Feinberg School of Medicine) observed that, similar to the general AIDS community ignoring neuroAIDS, the neuroAIDS community likewise ignores pediatrics. The biggest change in pediatric neuroAIDS has come from ART, which reduces mother-to-infant transmission-at least in the developed 
world. However, a number of questions remain unanswered in our response to pediatric neuroAIDS:

- Are we applying the available drugs optimally?

- In particular, when should we start therapy, given that both toxicity and pathogenesis may be different in a developing brain?

- How can we improve the drug development pipeline, which is too expensive (and thus too timid) to pursue neuroAIDS therapeutics aggressively?

\section{Discussion}

During the discussion that followed, one or more participants identified several important topics as deserving further investigation. These topics are summarized below in no particular order, other than the sequence in which they were raised during the discussion.

Does neuroAIDS progress in patients who are virally controlled? This begs the more basic question of whether peripheral or CNS virus is the reservoir during latency. A systematic study will be needed to answer these questions. However, troubling data have emerged from the CHARTER study suggesting that controlling the virus is not the same as eliminating the virus (or its effects) and that macrophages/microglia represent a major CNS reservoir of virus. Elyse Singer, University of California, Los Angeles, reported that she sees neurocognitive deficits in about half of her AIDS patients and that it seems to progress even with undetectable levels of CNS and plasma virus. However, there are no clinical guidelines for the treatment of neuroAIDS, and most clinicians do not even screen for it if they encounter peripheral neuropathy. If neurological disease does progress in virally controlled patients, the patients need to be informed.

Unique opportunities for neuroAIDS research in large US cities Immigration has created a single setting with small numbers of all possible populations-Why not study neuroAIDS in these populations in an industrialized setting? However, it is likely that investigators will find that the real problems in this group are not cognitive deficit, but gross psychiatric disease, complicated by IV drugs and other behavioral factors.

International research is vital There is no way to control the epidemic without trained personnel who can transfer existing knowledge as well as acquire new knowledge. In addition, the interaction of HIV and opportunistic infections cannot be properly studied in domestic settings. However, President's Emergency Plan for AIDS Relief (PEPFAR) has not really helped research, even international research. In general, NIH spends $10 \%$ of its AIDS budget on international activities. Should NIMH ask for new money or prioritize the available budget? What are the most important questions for international settings? Progression under ART? Migration of macrophages? Or the curious appearance of strokes and atherosclerosis among young HIV-1 seropositive men in Africa, a phenomenon that may not be unique to Africa?

Do more with available therapeutics Are there better ways to use existing ART to target the CNS and improve their effectiveness against neuroAIDS. Certainly, all new ART and adjuncts should be routinely screened for neuroAIDS indications. Some patients have a variety of cognitive and motor impairments, each of which has its own treatment regimen, some of which involve pretreatment with steroids. We need a more comprehensive, neuroprotective therapy for neuroAIDS that will preserve neurological function and quality of life.

NeuroAIDS is a bigger problem than we thought Current estimates of the incidence of HAND put it at 30\% of AIDS patients, which would make it the third most common dementia in the world. Good data are emerging from ACTG 5199 , but a more definitive study with standardized data will be needed to define global incidence.

Collect better data on neuroAIDS One participant suggested that neurocognitive categories should be added to the data collected by the International Epidemiologic Databases to Evaluate AIDS. This would require standardized measures to diagnose and quantify neurocognitive impacts. It would also be expensive; and while NIMH would like to make this change, it might be more effective to make better use of existing resources.

Involve the broader community and broaden the research focus NeuroAIDS does not just affect the patient; it also affects their caregivers and medical personnel. However, neurologists and psychiatrists are not dealing with neuroAIDS, despite its many commonalities with Alzheimer's and multiple sclerosis. There may be clues in the successful treatments of those diseases. By the same token, how can the study of neuroAIDS inform the study of other neurodegenerative diseases? Will it be possible to develop standardized measures, assays, and imaging protocols?

Patients do not die from neuroAIDS Instead, they die from opportunistic infections (OIs), especially internationally, often before they have time to become demented. In addition, OIs add significantly to the cost of treating AIDS patients. Ideally, we should treat HAND and neurological 
OIs simultaneously. The low-hanging fruit in this area is toxoplasmosis encephalitis, the most common neurological condition in HIV-1-seropositive patients in the ART era. Several therapies are available in the USA, at widely varying prices; but cheaper alternatives will be needed to address this problem in the rest of the world.

We may be seeing a paradigm shift in the study of neuroAIDS Past research has focused narrowly on the virus, and as a result, all available therapeutics target the virus. NeuroAIDS would now appear to be a broader and more complex process of immune dysregulation that involves multiple systems. As a result, neuroAIDS researchers will need to think more globally, in terms of reservoirs, other cell types, neurodevelopment, and other drug targets. To do this, they will need to bring additional scientific disciplines to bear on neuroAIDS and put together interdisciplinary research teams.

Develop better animal models of neuroAIDS Several participants suggested that current animal models do not accurately portray the current, clinical experience of viral control, and chronic inflammation under ART. In SIV, early events determine the neurological outcome, but the virus is in the brain early and death sometimes follows within 30 days. The endotoxemic mouse may be a better (or less expensive) model of chronic CNS inflammation. There is a special need for animal studies to determine when to initiate neuroprotective therapy, as well as for vaccine studies, and in a few years, there will be a demand for additional animals to test the in vivo efficacy of the numerous HIV protein antagonists that will emerge from current research.

Standardization will enhance collaboration NIH and others have invested millions of dollars in developing extensive data sets and specimen collections; but while the cohorts must remain separate, there is no need for the data and specimens to remain separate. NIMH should do more to ensure that data collection produces a research set. It should also support efforts to harmonize and standardize the definitions, categories, measures, assays, and protocols that are used by different collaborations; encourage those collaborations to share data and compile catalogs of their specimen collections; and to make these resources available to outside investigators. One participant suggested that a good initiative in this direction would be for NIMH, NINDS, and NIDA to convene a consensus conference or to sponsor a consensus paper to set out the components of a standard neurological evaluation for HIV patients. Another participant said that this would take 5 to 7 years to get into the scientific literature and suggested that NIH could make it so tomorrow, by merely promulgating the standard itself. The NIH standards could take the form of a "tool box" that identifies all of the validated tests that are available for evaluating the cognitive, verbal, and motor abilities of neuroAIDS patients.

Follow-up of well-characterized cohorts Uncertainty about the progression of HAND during HAART can be addressed through careful tracking of patient cohorts. Natural history (ALLRT, CHAVI, CHARTER) and neuroprotective clinical trial cohorts (ACTG) implement validated clinical, neuropsychologic, and virologic tests that are critical for assessing long-term outcomes. Short-term studies (months) are unlikely to yield definitive conclusions about the effectiveness of neuroprotective strategies in a chronic syndrome such as HAND. Long-term outcomes (years) must be determined in such carefully characterized cohorts.

Involving the vaccine community in CNS studies Vaccine testing in the macaque SIV models provides valuable information about effectiveness of immune-mediated systemic viral load suppression on immunopathogenesis and survival. Similar CNS studies in vaccine cohorts are critical to determine the neuroprotective potential of effective vaccines. Macaque vaccine studies produce tissue specimens and laboratory data that can strongly support correlative and outcome-based studies of CNS pathogenesis. Systematic analyses of CNS tissues in these animals should be aggressively pursued. The CHAVI study of innate immunity markers (120 acute HIV infections/AHI) is defining the kinetics of systemic elevations in acute-phase proteins, cytokines, and chemokines during the earliest stages of infection (transmission, viral eclipse phase). The frequencies and function of peripheral DC, NK, and NKT cells and the kinetics of antibody production are also being examined. These early events could influence CNS invasion, neuroimmune responses, and cognitive outcomes.

What early events influence CNS outcomes? This is among the most difficult questions to answer and it requires longitudinal analyses of recent seroconverters (CHAVI). Functional brain imaging is critical for defining early brain responses to systemic and CNS infection because it is the only method capable of analyzing brain metabolism in real time. However, the necessary technology for such studies is not easily transferable in limited resource settings. Acute HIV infection serological markers of innate immunity (IB) should be examined as associative and predictive markers for neuropathogenesis.

Host responses in the CNS Along with analysis of CNS neurochemical responses by functional imaging, cerebrospinal fluid sampling and analysis are also essential. Long-term follow-up of carefully characterized cohorts (e.g., CHAVI seroconverters) is essential; short-term cohort studies repre- 
sent a relatively inefficient use of resources. This will determine whether targeted neuroprotection can or should be applied in chronically infected individuals.

When should treatment be initiated? Current criteria for initiation of ART do not consider CNS outcomes as a benchmark of efficacy. Establishing CNS-based criteria for initiation of therapy should be the ultimate goal of neuroprotection strategies. This applies to ART as well as candidate adjunctive neuroprotective drugs as they are developed. Determining neurological outcomes in acute seroconverters (ART treated and untreated) is the critical first step.

Opportunistic infections-optimal and cost-effective treatment The diagnostic criteria will depend on standard laboratory diagnostic tests and cost-effective brain imaging. Preliminary studies support OIs as significant contributors to the morbidity of AIDS in underdeveloped countries. The first priority should be determination of the prevalence of CNS OIs (TB, toxoplasmosis, crytptococcus).

Other complications (i.e., vascular) The effects of HIV and ART accelerate the risk of cardiovascular disease, but the risk of cerebrovascular disease (CVD) and its complications is undefined. Recent studies within the USA suggest an increased risk of CVD, and investigating this risk in patients in underdeveloped countries requires technology transfer and infrastructure development.

Influence of cellular entry on the development of CNS infection The SIV macaque models most closely mimic
HIV infection in humans. Both acute and chronic CNS pathogenesis models are useful, and emphasis should be placed on developing antiretroviral drug treatment paradigms that mimic ART regimens and that alter disease course. Defining sources and adequate supplies of drugs is a priority. Other relevant models should be explored but realistically, to date none have fully modeled the steps from HIV neuroinvasion through neurodegeneration.

\section{Concluding remarks}

The importance of neuroAIDS remains significant to the HIV/AIDS problem. This series of discussions and priority sharing have served to bridge needed research to outline a plan for growth and development of the field and to tackle critical problems overall. These priorities and ideas provide a template for future growth of the field and its long-term impact.

Acknowledgments The authors wish to acknowledge the National Institute of Mental Health (NIMH), National Institute on Drug Abuse (NIDA), and National Institute of Neurological Disorders and Stroke (NINDS) for sponsorship and research support. We wish to thank science writer Paul Phelps for organizing the results from this meeting. Also, we thank Dr. R. Lee Mosley and Robin Taylor, University of Nebraska Medical Center for the critical review and active discussions of the article.

Open Access This article is distributed under the terms of the Creative Commons Attribution Noncommercial License which permits any noncommercial use, distribution, and reproduction in any medium, provided the original author(s) and source are credited. 